\title{
Correction to: Automatic virtual reconstruction of maxillofacial bone defects assisted by ICP (iterative closest point) algorithm and normal people database
}

\author{
Bimeng Jie ${ }^{1,2} \cdot$ Boxuan $\mathrm{Han}^{3} \cdot$ Baocheng Yao ${ }^{1,2} \cdot \mathrm{Yi} \mathrm{Zhang}^{1,2} \cdot$ Hongen Liao ${ }^{3} \cdot \mathrm{Yang} \mathrm{He}^{1,2}$
}

Published online: 27 November 2021

○) Springer-Verlag GmbH Germany, part of Springer Nature 2021

\section{Correction to: Clinical Oral Investigations}

https://doi.org/10.1007/s00784-021-04181-3

In the article entitled "Automatic virtual reconstruction of maxillofacial bone defects assisted by ICP (iterative closest point) algorithm and normal people database, by Jie et al.,

This is an official statement of erratum made by authors for better understanding of readers Figs. 3, 4, 5, 6, 7 and 8 . Specific corrections were as follows:

there was error in the Figures and Funding.

Fig. 3 Protocol of model

experiment

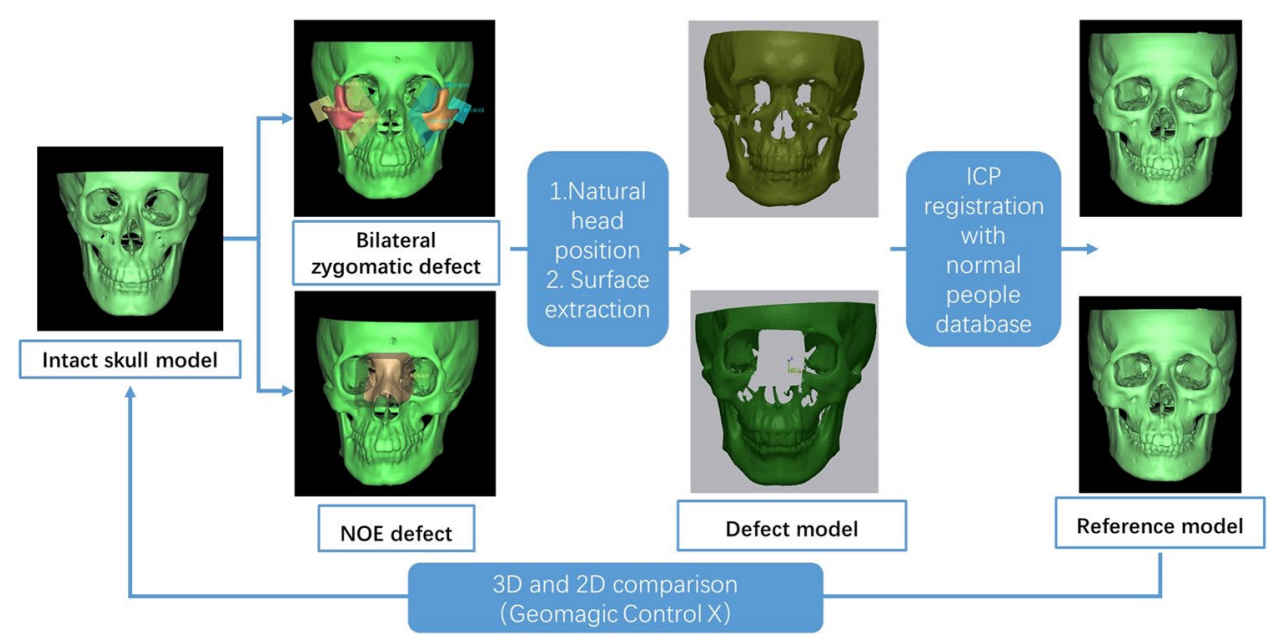

The original article can be found online at https://doi.org/10.1007/ s00784-021-04181-3.

\section{Hongen Liao}

liao@tsinghua.edu.cn

$\triangle$ Yang He

fridaydust1983@163.com

1 Department of Oral and Maxillofacial Surgery, Peking University School and Hospital of Stomatology,

22 Zhongguancun South Road, Beijing 100081,

People's Republic of China
2 National Engineering Laboratory for Digital and Material Technology of Stomatology, Beijing Key Laboratory of Digital Stomatology, National Clinical Research Center for Oral Diseases, Beijing, People's Republic of China

3 Department of Biomedical Engineering, School of Medicine, Tsinghua University, Beijing 100084 , People's Republic of China 
A

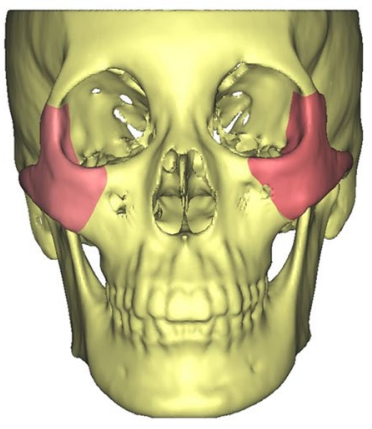

B

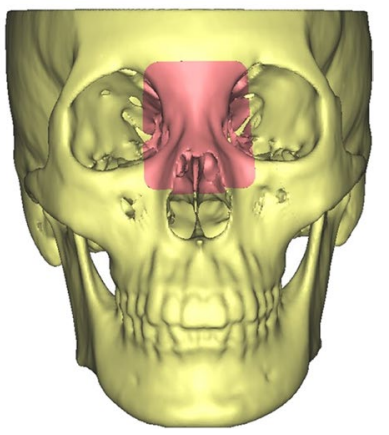

Fig. 4 Artificial defects of bilateral zygomatic and naso-orbitalethmoid (NOE) regions. A Virtual osteotomy planes passing through bilateral zygomaticomaxillary suture, zygomaticofrontal suture, and root of the zygomatic arch. B Virtual osteotomy planes passing through the bilateral Fso and Or points

Fig. 5 3D comparison of the defect area.A Bilateral zygomatic area of S1r. B NOE area of $\mathrm{S} 2 \mathrm{r}$

Fig. 6 2D comparison of the bilateral zygomatic area.A Section plane parallel to the Frankfort plane passing through the bilateral Mp point.B RMSE of the outer curve and deviation of $\mathrm{Mp}$ and $\mathrm{Zy}$ points between $\mathrm{S} 1 \mathrm{r}$ and $\mathrm{S} 0$ were calculated

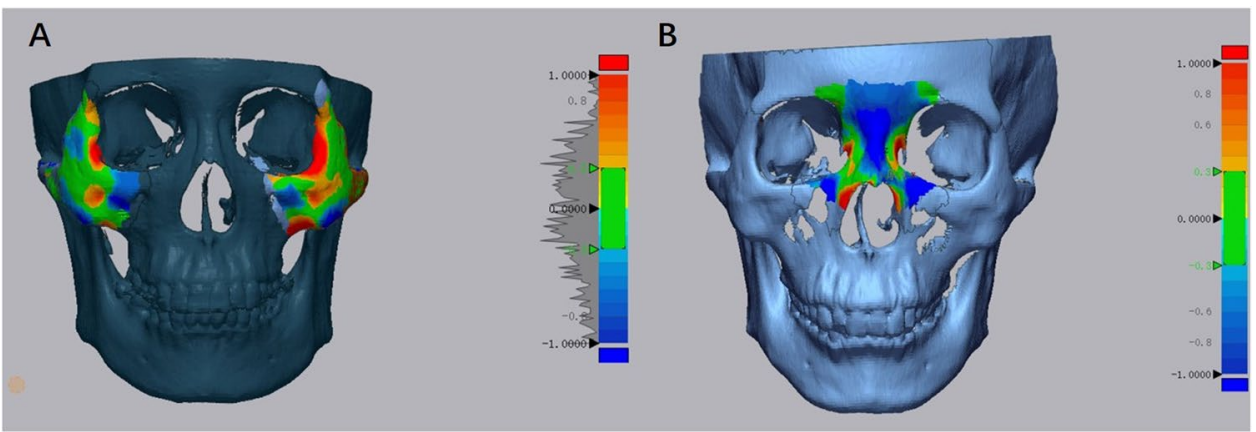

A

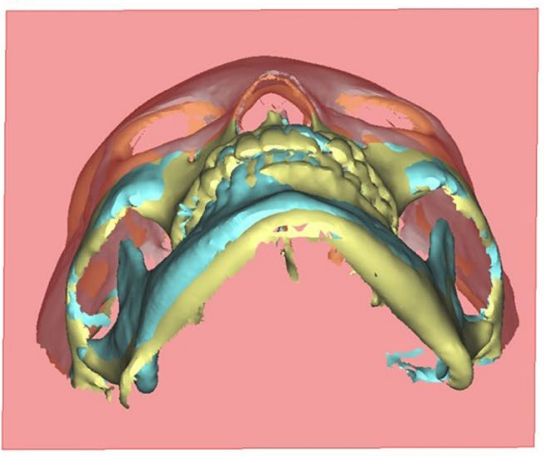

B
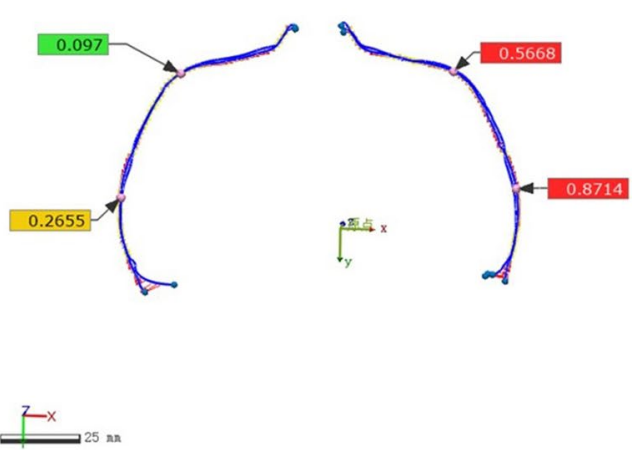
Fig. 7 2D comparison of the NOE area.A Mid-sagittal section plane and deviation of glabella, nasion, and rhinion point. B Axial section plane and deviation of bilateral inner canthal ligament (ICL)

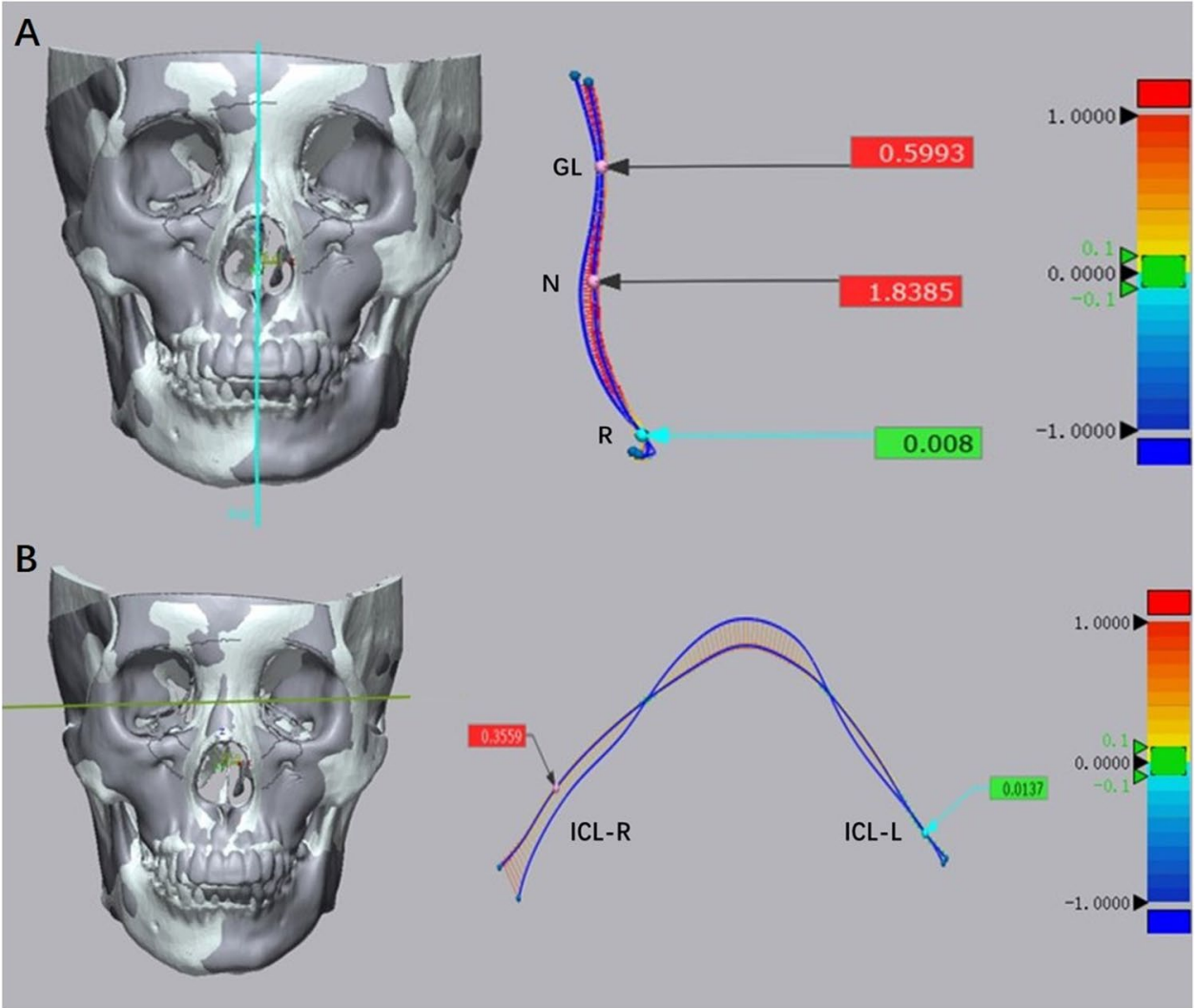


Fig. 8 A representative case of patient No. 3. A Pre-operative frontal view. B Pre-operative lateral view. C Pre-operative panorama showing lesion of anterior mandible.DVirtual tumor resection (red). $\mathbf{E}$

Reference data and automated completion of defect (green).F Twophase DCIA flap reconstruction plan.G-H Tumor resection under guidance of surgical template.I-J Flap positioning under guidance of prebent titanium plate and surgical template.K One week postoperative skull reconstructed from CT data.L Mirroring of the postoperative skull.M 3D comparison of post-operative skull (yellow) and its mirrored model (blue).N-O 6 months postoperative photograph showing satisfying appearance
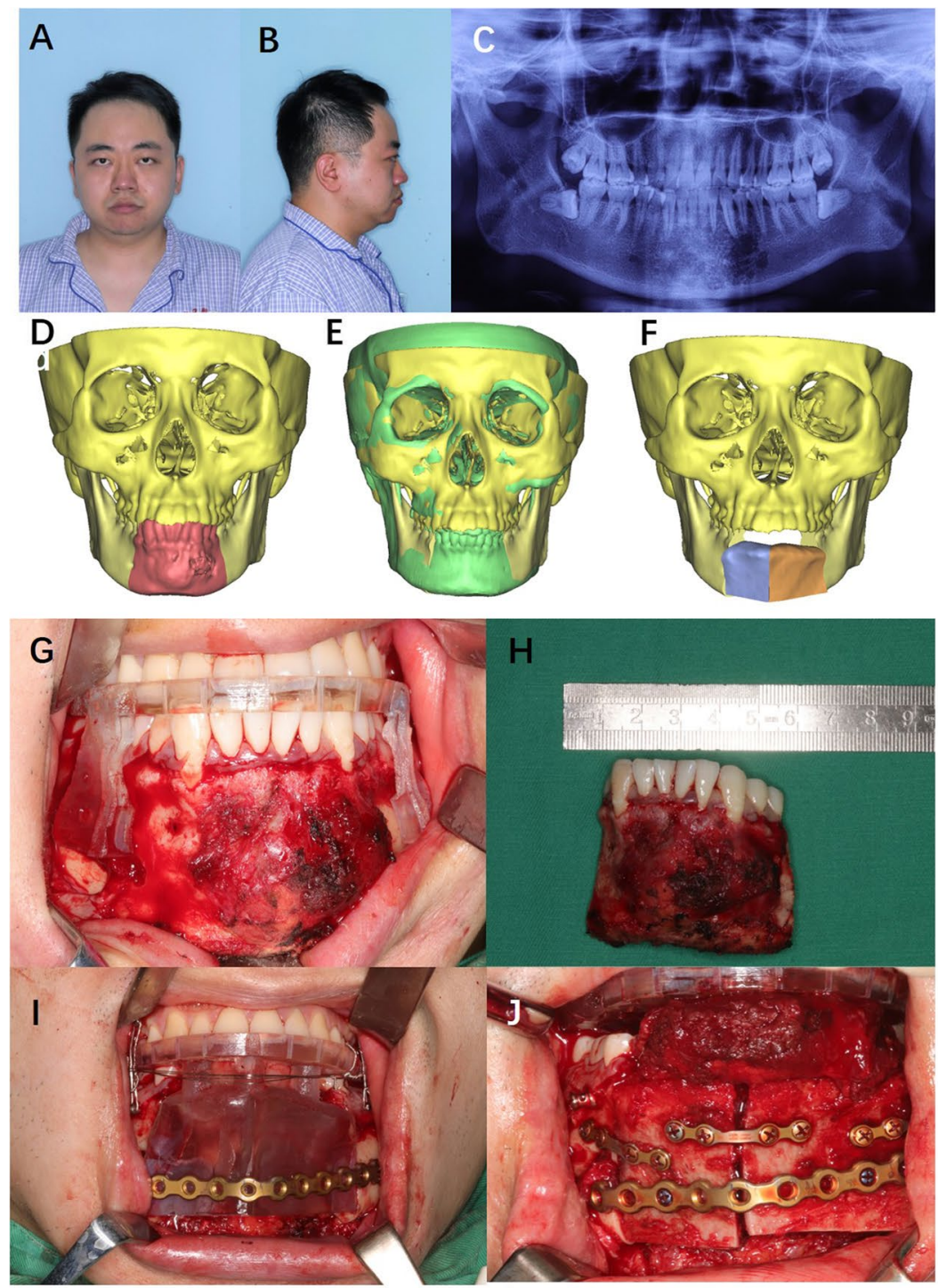

$\mathrm{K}$

$\mathrm{L}$
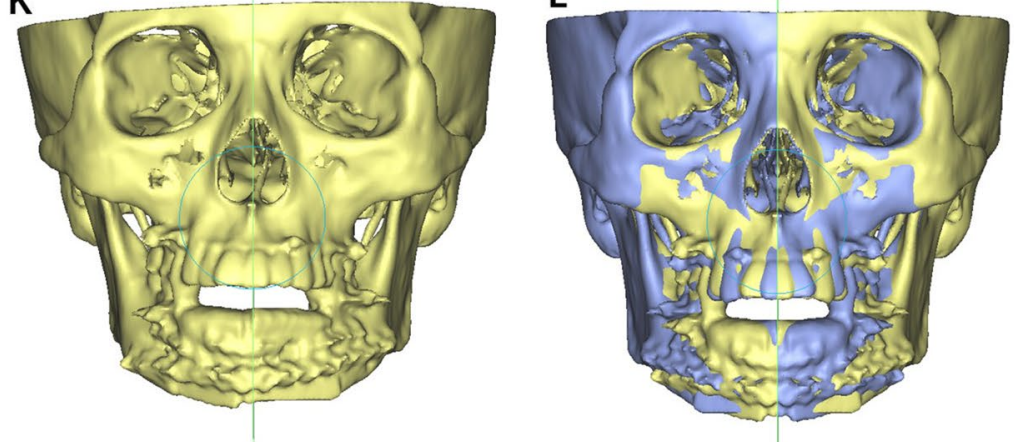
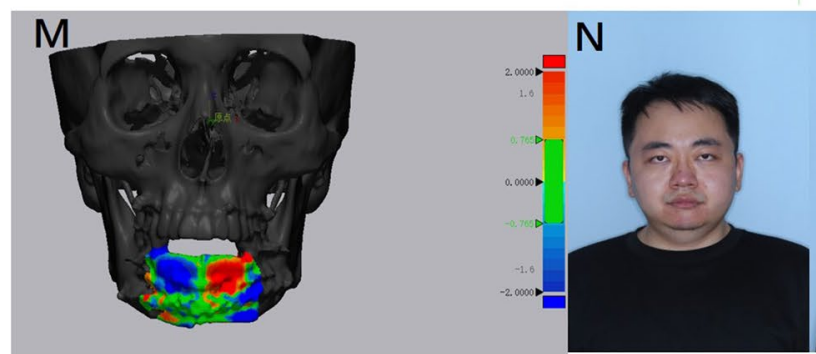

O

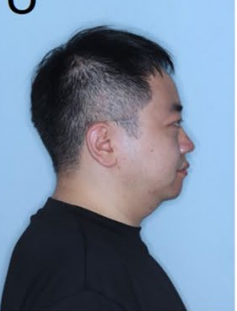


The original article has been corrected.

Funding This study was supported by National Key Research and Development Program of China (grant No. 2019YFF0302401\&2017YFB1104103), National Natural Science Foundation of China (grant No. 82027807\&81771940) and Beijing Municipal Natural Science Foundation (7212202).
Publisher's note Springer Nature remains neutral with regard to jurisdictional claims in published maps and institutional affiliations. 